\title{
PRUEBA DE RIGIDEZ DIELÉCTRICA A ELEMENTOS DE PROTECCIÓN PARA TRABAJOS CON TENSIÓN TCT
}

\section{Dielectric strength test to protection elements for live lines works}

\author{
Carlos Eduardo Pinto-Salamanca ${ }^{1}$, Juan Carlos Castro-Galeano ${ }^{2}$ \\ ${ }^{1}$ Ing., Universidad Pedagógica y Tecnológica de Colombia (Duitama, Boyacá, Colombia).carlos.pinto@uptc.edu.co \\ ${ }^{2}$ MSc., Universidad Pedagógica y Tecnológica de Colombia (Duitama-Boyacá, Colombia). juan.castrogaleano@uptc.edu.co
}

(Recibido noviembre 23 de 2016 y Aprobado junio 5 de 2017)

\begin{abstract}
Resumen
Este artículo presenta el diseño y montaje de un sistema de pruebas de voltaje sostenido a elementos y equipos empleados en maniobras de línea viva, a través de ensayos a guantes y pértigas, pues son los primeros puntos de contacto que garantizan operaciones seguras. Esto significa un avance para la creación de un laboratorio certificado en pruebas de este tipo en la Universidad Pedagógica y Tecnológica de Colombia UPTC, Facultad Seccional Duitama, si se considera que actualmente no se cuenta con laboratorios que presten el servicio en la región de Boyacá y Casanare. Se establecieron los ensayos de rigidez dieléctrica a los elementos y equipos de protección personal bajo los parámetros de las normas ASTM D120, ASTM F496, ISO 60903, ASTM-F711 y la IEEE 978, desarrollando un montaje para la realización de pruebas a guantes y pértigas con niveles de tensión de hasta $15 \mathrm{kV}$. Los resultados validan el sistema propuesto desde el punto de vista del diseño y la implementación del circuito, donde se hicieron pruebas para establecer capacidades dieléctricas, cuando funcionaban en condiciones de circuito abierto, con carga resistiva o en corto circuito. Se verificó el cumplimiento de la normatividad establecida bajo las secuencias de prueba de parámetros de seguridad para el sistema y el seguimiento a los ensayos hechos, mediante el uso de un sistema de gestión para la generación de conceptos de aprobación o rechazo de los elementos probados.
\end{abstract}

Palabras clave: rigidez dieléctrica; trabajos con tensión (TCT); voltaje sostenido.

\begin{abstract}
This paper presents the design and assembly of a system of tests of sustained voltage to elements and equipment used in live line maneuvers through tests on gloves and dielectric rods, as these are the first points of contact to ensure safe operations. It means an advance for the creation of a laboratory certified in this type of tests at Universidad Pedagógica y Tecnológica de Colombia (UPTC) Faculty of Duitama, considering that currently there are not laboratories that provide this service in Boyacá and Casanare. Dielectric strength tests were performed on personal protection elements and equipment under the parameters of ASTM D120, ASTM F496, ISO 60903, ASTM-F711 and IEEE 978, developing an assembly for testing gloves and dielectric rods with voltage levels up to $15 \mathrm{kV}$. The results validate the proposed system to outlook of circuit design and implementation, where tests were performed to establish dielectric capacities, in operating under open circuit conditions, with resistive load or short circuit. The compliance with the regulations established under the test sequences of safety parameters for the system and the follow-up to the tests was verified through the use of a management system for the generation of concepts of approval or rejection of the tested elements.
\end{abstract}

Keywords: dielectric strength; works with voltage (JWV); sustained voltage.

\section{INTRODUCCIÓN}

En el sistema eléctrico de potencia (SEP) es indispensable la realización de tareas de mantenimiento, ampliación y renovación de líneas de transmisión y distribución, para disponer de forma eficiente de un servicio continuo y sin posibles fallas. Estas tareas pueden hacerse en redes desenergizadas (sacando de servicio los circuitos) o en

Cómo citar este artículo:

C.E. Pinto-Salamanca y J.C. Castro-Galeano, "Prueba de rigidez dieléctrica a elementos de protección para trabajos con tensión TCT", Revista Ingeniería Investigación y Desarrollo, vol. 17 N², pp. 83-92, Julio, 2017. 
redes energizadas, también denominada de "línea viva" o trabajos con tensión TCT, los cuales se desarrollan con el objetivo de no sacar de servicio los circuitos para no afectar la continuidad, eficiencia y confiabilidad de sistema.

Existen distintas forma de denominar los trabajos en redes energizadas, las más usadas son TCT trabajos con tensión y línea viva, que consiste en la ejecución de trabajos de mantenimiento en redes energizadas con niveles de tensión hasta 800 kV (500 kV máximos para Colombia actualmente), sin suspender el suministro de energía. Según el Reglamento Técnico para Instalaciones de Redes Eléctricas -RETIE- [1], línea viva es un término aplicado a una línea con tensión o una línea energizada para arreglar fallas, ampliar la red, cambiar postes, entre otros.

Las operaciones en TCT implican altos estándares de seguridad para la protección de la vida de los operarios y de los mismos componentes del sistema, ya que los riesgos a los que se somete un operador de línea viva son considerables, debido a que está expuesto a descargas de alta tensión y elevados niveles de corriente, que pueden generar daños tan graves como lesiones, quemaduras, caídas de altura o incluso la muerte.

En Colombia, la Comisión de Regulación de Energía y Gas -CREG- [2] establece la responsabilidad del operador de red (OR) regional en la operación, mantenimiento y expansión del sistema de distribución local de energía eléctrica, definiendo sanciones y multas como el "cargo por confiabilidad", a las empresas del sector eléctrico que dejen sin servicio a los usuarios que dependen del mismo. Por su parte, el Ministerio de la Protección Social define, mediante la Resolución 1348 de 2009 [3], el reglamento de salud ocupacional para procesos de generación, transmisión y distribución de energía eléctrica en las empresas del sector eléctrico, estableciendo que los procedimientos, equipos y materiales utilizados en el método de trabajo para que el trabajador no entre en contacto accidentalmente con cualquier otro elemento o potencial distinto al suyo

Los operarios que ejecutan el trabajo en línea viva necesitan un equipo especial de protección personal. Estos elementos deben fabricarse con materiales sintéticos de alta resistencia al efecto corona, en colores establecidos según normatividad. Lo anterior requiere del desarrollo de procesos de certificación posteriores a la fabricación de los elementos de protección y, por ende, establece una demanda de laboratorios que realicen ensayos dieléctricos, que deben estar acreditados por el ONAC [4] bajo el estándar NTC ISO-17025 [5]. Se indica que se deben certificar los laboratorios, el laboratorista y la prueba por realizar.

\section{PRUEBAS DIELÉCTRICAS PARA GUANTES Y PÉRTIGAS}

Gran parte de los trabajos de mantenimiento de redes eléctricas aéreas de distribución de línea viva emplean técnicas de trabajo a contacto, mediante el uso de equipo de protección flexible, fabricado con materiales de tipo caucho natural, compuestos elastómeros o, recientemente, por fibras sintéticas con diferentes grados de flexibilidad y resistencia al envejecimiento, a la alteración de sus características por fenómenos atmosféricos y a la resistencia al efecto corona.

Los guantes dieléctricos son designados como Tipo I o Tipo II y en cada caso subdivididos como clase 00 , $0,1,2,3$ y 4 . La designación de tipo se determina por las normas ASTM D 120[6] y UNE-EN 60903 de 2005[7]: Tipo I (no resisten al ozono, fabricados con un alto grado compuesto CIS-1,4-poliisopreno de origen natural o sintético, vulcanizado adecuadamente); Tipo II (resistente al ozono, hecho de cualquier elastómero o combinación de compuestos de elastómero).

Las pértigas dieléctricas son fabricadas en resina epóxica y se usan en TCT bajo la técnica a distancia, para múltiples tareas a la hora de hacer trabajos de mantenimiento y ampliación del sistema, como los siguientes: apertura o cierre de seccionadores, instalación de equipos de puesta a tierra, verificación de existencia de tensión por acople de detectores, tijeras de corte, perfiladores, entre otros. También pueden usarse en combinación con las demás técnicas de trabajos en línea viva y no son aptas para permanecer bajo tensión durante períodos de tiempo prolongados $[17,18]$.

Las pértigas aislantes están constituidas por tubos de resina epóxica, reforzados con fibra de vidrio y rellenos de espuma de poliuretano. Las pértigas se clasifican en tres, telescópicas, rígidas o acoplables y tipo escopeta o de gancho retráctil. Las dimensiones de las pértigas comerciales se prueban hasta $75 \mathrm{kV}$ y tienen una longitud máxima de 12,2 m que es la pértiga telescópica de 8 secciones. 


\subsection{Prueba de voltaje sostenido en guantes}

Se denomina prueba de voltaje sostenido [8] al ensayo que se realiza a los guantes, sometiéndolos a una diferencia de potencial que se eleva hasta su nivel de tensión de prueba, manteniéndolo durante un tiempo determinado de 1 minuto. Este tipo de prueba supone una inspección previa de las propiedades físicas del guante, verificando que no tenga fisuras, quemaduras, porosidades, entre otros aspectos que si se detectan desde el comienzo, ya indican que debe ser rechazado.

A partir de lo establecido en la norma ASTM D 120 artículo 18 , se presenta la prueba de voltaje sostenido, teniendo en cuenta que la tensión debe subir de manera controlada por medio de un variac, a razón de $1000 \mathrm{~V} / \mathrm{s}$ hasta la tensión permitida según la clase del guante. Todas las pruebas se harán a temperatura ambiente, los guantes se llenarán con agua del grifo y se sumergirán en agua a una profundidad considerando un espacio libre en el mango del guante. El agua, durante el ensayo, deberá tener las mismas características químicas dentro y fuera del guante, además debe estar libre de burbujas y bolsas de aire, y la parte expuesta del guante por encima de la línea de agua deberá estar seca. El agua, dentro del guante, deberá tener un electrodo que estará conectado a un terminal de la fuente de tensión, ya sea en forma de varilla, cadena móvil o deslizante dentro del agua. El agua, fuera del guante, deberá tener contacto con el otro terminal de la fuente de tensión. Los valores de corriente permitida se muestran en la Tabla 1, mientras que los valores de tensión de prueba en DC y en AC se muestran en las tablas 2 y 3.

Tabla 1. Corriente permitida en la prueba

\begin{tabular}{ccccc}
\hline \multicolumn{5}{c}{ Máxima corriente de prueba [mArms] / } \\
TALANA GE & \multicolumn{5}{c}{ GANTE } \\
& $\begin{array}{c}280 \\
{[\mathrm{~mm}]}\end{array}$ & $360[\mathrm{~mm}]$ & $410[\mathrm{~mm}]$ & $\begin{array}{c}460 \\
{[\mathrm{~mm}]}\end{array}$ \\
\hline 00 & 8 & 12 & No aplica & No aplica \\
0 & 8 & 12 & 14 & 16 \\
1 & No aplica & 14 & 16 & 18 \\
2 & No aplica & 16 & 18 & 20 \\
3 & No aplica & 18 & 20 & 22 \\
4 & No aplica & No aplica & 22 & 24 \\
\hline
\end{tabular}

Tabla 2. Voltaje de prueba en DC

\begin{tabular}{ccc}
$\begin{array}{c}\text { CLASE DE } \\
\text { GUANTE }\end{array}$ & $\begin{array}{c}\text { VOLTAJE PROMEDIO } \\
\text { DE PRUEBA [V] }\end{array}$ & $\begin{array}{c}\text { VOLTAJE PROMEDIO DE } \\
\text { RUPTURA (DESGLOCE) } \\
\text { [V] }\end{array}$ \\
\hline 00 & 10000 & 13000 \\
0 & 20000 & 35000 \\
1 & 40000 & 60000 \\
2 & 50000 & 70000 \\
3 & 60000 & 80000 \\
4 & 70000 & 90000 \\
\hline
\end{tabular}

Tabla 3. Voltaje de prueba en AC

\begin{tabular}{ccc}
\hline $\begin{array}{c}\text { CLASE DE } \\
\text { GUANTE }\end{array}$ & $\begin{array}{c}\text { VOLTAJE DE } \\
\text { PRUEBA [VRMS] }\end{array}$ & $\begin{array}{c}\text { VOLTAJE DE RUPTURA } \\
\text { (Breackdown) }\end{array}$ \\
\hline 00 & 2500 & 4000 \\
[VRMS]
\end{tabular}

En el método de tensión sostenida se pueden realizar pruebas con tensión alterna o continua, en función de la selección del propietario de los elementos. El equipo de prueba utilizado en el ensayo de ruptura dieléctrica deberá suministrar corriente continuamente o de forma variable durante el ensayo.

En la pruebas de (AC) como en (DC), cada guante deberá someterse a la verificación de conformidad con los requisitos y secuencias de las normas ASTM D120 e ISO 60903.

\subsection{Prueba de voltaje sostenido en pértigas}

Los ensayos de pértigas se basan en las normas IEEE 978 de 1984 [9] y la norma ASTM F 711 [10], además de las especificaciones técnicas $01.20 / 0$ para pértigas telescópicas, y 01.19/0 para pértigas de maniobra para MT. Las normas IEC 855 de1985 [11], IEC 832 de 1988 [12], IEC 410 de 1973 [13] permiten también definir un procedimiento para la realización de las pruebas sin alterar su forma y sus propiedades. 
La prueba consiste en generar un nivel de tensión de manera controlada a partir de los electrodos del transformador a los dos tubos conductores que estarán anclados en el mismo lugar donde está soportada la pértiga, la cual se someterá a una diferencia de tensión en puntos cercanos, y según la Norma IEEE 978 de 1984, se establece que sean hasta $75 \mathrm{kV}$ los que entregue el transformador en dos puntos a una distancia de $30 \mathrm{~cm}$ entre ellos.

La tensión de prueba se puede ir incrementando o puede cambiar de manera instantánea o súbita, de modo que no afecte las propiedades físicas de los elementos que constituyen la pértiga, debido a que estas herramientas son para trabajos que no sean prolongados. Los tubos conductores permiten el paso de la energía a las pértigas por medio de cadenas que sujetan a la pértiga con resortes, como se muestra en la Figura 14 y se estipula en la norma IEEE 978 de 1984 y ASTM F 711. Estos valores de tensión son únicamente para equipos de AC.

Para hacer la prueba con corriente continua, los niveles son superiores. Los ensayos de tipo contemplan la prueba dieléctrica, mediante las normas IEC 855 y en IEC 832, donde se estipulan los materiales que se usan para la construcción de la pértiga y, por ende, las propiedades dieléctricas pertinentes del material.

\section{DISEÑO Y CONTRUCCIÓN DEL SISTEMA DE PRUEBAS DIELÉCTRICAS}

El sistema propuesto consiste en la aplicación de tensión durante un tiempo determinado a guantes $y$ a pértigas hasta $15 \mathrm{kV}$ en $\mathrm{AC}$, que permite desarrollar pruebas de aceptación de la continuidad del uso de guantes o pértigas, para generar un concepto de aprobación o rechazo de los elementos por probar. Se determina si el aislamiento del elemento se encuentra en condiciones óptimas para el desarrollo de tareas en TCT. Con el fin de establecer si el aislamiento de los elementos de seguridad es óptimo en TCT, se propuso un equipamiento para realizar pruebas según el sistema descrito en la Figura 1, que está compuesto por:

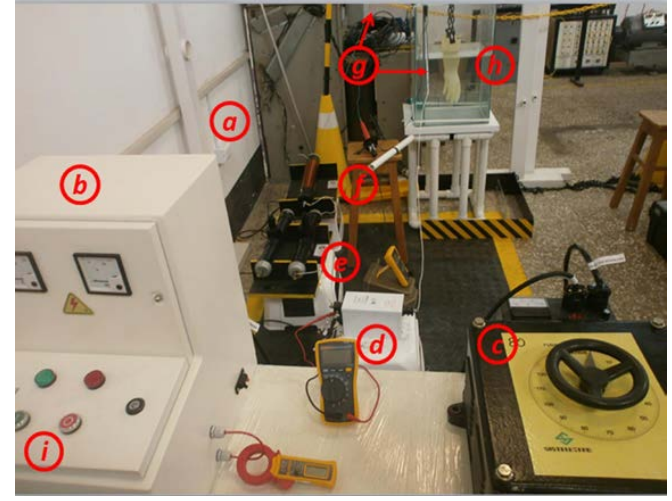

Figura 1. Componentes del sistema de pruebas

a. Conexión a la red eléctrica monofásica 120 Vrms.

b. Tablero de control, que permite dar inicio a la prueba y consta de elementos de medición como amperímetro de 0 -5 A y voltímetro 0-120V, además de pulsadores, transformador de corriente de 30-5 A, transformador de tensión, fusible y portafusible de $5 \mathrm{~A}$, entre otros. Además sirve como mesa para el variador de tensión y soporte de voltímetro y amperímetro en baja tensión

c. Variac monofásico (0-140 V), que permite cambios controlados de la tensión de prueba, y fija el valor al cual se quiere llegar en baja tensión.

d. Transformador elevador de tensión (120-13500 V) relación de transformación de 125: suministra la tensión correspondiente a los tipos de elementos, teniendo en cuenta la clasificación de los mismos. A partir de los $1000 \mathrm{msnm}$, el transformador disminuye su aislamiento en $1 \%$ por cada $100 \mathrm{~m}$ de elevación, afectando sus componentes y disminuyendo de manera significativa el porcentaje de la tensión de salida para su correcto funcionamiento. Para el caso específico de la ubicación en Duitama, Boyacá ( $2530 \mathrm{~m}$ sobre el nivel del mar) se dispone del $84 \%$ del voltaje de salida nominal. Se usó un transformador elevador empleado en lámparas de neón, de $15 \mathrm{kV}$, reduciendo su capacidad a $12.6 \mathrm{kV}$ $(15 \mathrm{kVx} 0.84)$ 
e. Resistencia limitadora para manejar las sobretensiones o sobrecorrientes generadas si el guante es perforado. La resistividad depende del agua que se use, se encuentra en un rango de $500 \mathrm{k} \Omega$ a $1 \mathrm{M} \Omega$, debido a que el valor total de la resistencia limitadora se calculó considerando las máximas corrientes en el lado de alta tensión del transformador de $30 \mathrm{~mA}$ y en caso de ser la única carga significativa en el circuito manifestaría una disipación de potencia de 450VA

f. Bobina de inducción para regular y disminuir armónicos, transitorios y agentes eléctricos que alteren la red que suministra la energía eléctrica. Debido a los cambios bruscos de corriente que se presentan en una falla del elemento de pruebas, se decidió colocar una impedancia que amortigüe este fenómeno y que, de esta manera, proteja los elementos del sistema y no afecte los aparatos que están conectados a la red. La bobina fue construida con alambre de cobre calibre 24 AWG, con una impedancia de 11,3445 Mh.

g. Soporte para elementos por ensayar, cerrando el circuito mediante los terminales 1 y 2 , como electrodos que permiten el paso de corriente a los elementos por probar.

h. Para el caso de ensayos a guantes, el soporte está compuesto por una cubeta de pruebas que permite sumergir en agua el elemento de prueba (ver Figura 2). Para las pruebas en pértigas, los electrodos difieren en la conexión de las terminales 1 y 2 que van a los tubos conductores que se encuentran anclados a los soportes y conexión por resorte construidos según la normatividad (ver Figura 3).

i. Adicionalmente, en el sistema de pruebas se establecen unas consideraciones técnicas con el fin de garantizar un ensayo seguro. Lo cual se contempló en el tablero de control que energiza o desenergiza el circuito de pruebas, teniendo en cuenta los niveles de corriente que pueden presentarse en condiciones normales o de falla.

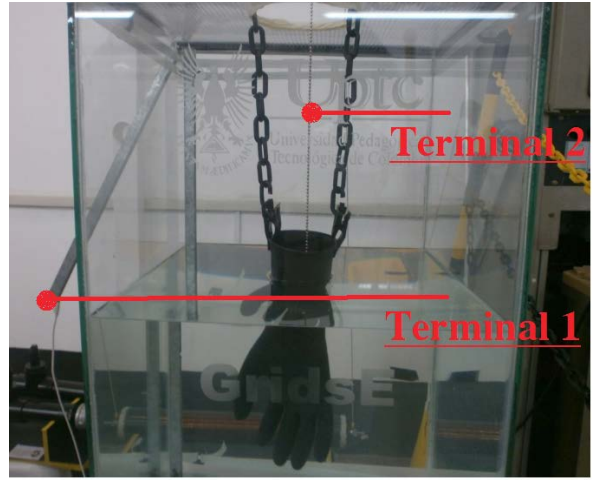

Figura 2. Cubeta de pruebas

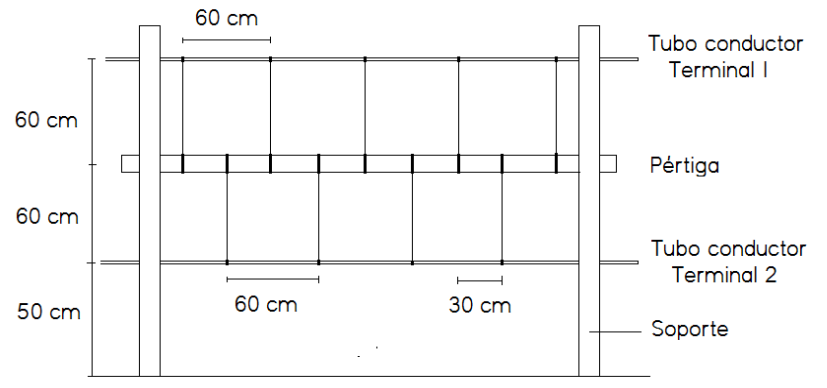

Figura 3. Soporte para pértigas

Además del equipamiento de pruebas, se elaboró un sistema de gestión de resultados para documentar los ensayos, fijando los niveles de tensión que se usan, dependiendo del objeto que vaya a ser sometido a pruebas y que permita generar un reporte.

Para la seguridad de los operarios del sistema de prueba, se implementó una señalización visible de la zona de trabajo (ver Figura 4), así como implementos utilizados para realizar los ensayos, lo que implica la capacitación adecuada para el uso de los elementos requeridos. En el tablero de control, una señal luminosa indica cuando el sistema está energizado y otra cuando se inicia la aplicación de tensión. 


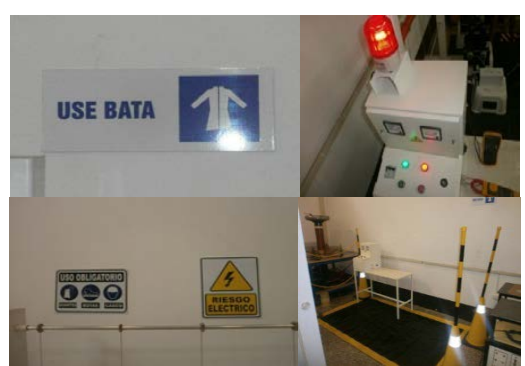

Figura 4. Señalización de área de trabajo e implementos para usar.

Tanto para las pruebas a guantes como a pértigas, el circuito de pruebas de alta tensión requiere de los siguientes elementos: tablero de control, variac, transformador elevador, resistencias limitadoras, reactancia inductiva y soportes, tal como se muestra en la Figura 5.

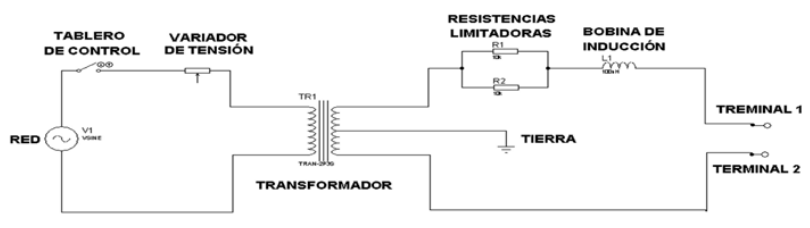

Figura 5. Esquema de pruebas. Guantes y pértigas.

\section{VERIFICACIÓN Y APLICACIÓN DE LA SECUENCIA DE PRUEBA}

Las pruebas disponibles en el sistema propuesto en este proyecto fueron diseñadas con fines académicos e industriales, mediante la técnica de tensión sostenida a guantes y pértigas hasta $15 \mathrm{kV}$ en $(\mathrm{AC})$, considerando a estos como los principales elementos que están en contacto con las líneas de transmisión y redes de distribución en su correspondiente técnica de trabajo.

La prueba de voltaje sostenido consiste en alimentar con una tensión variable los implementos de protección personal indicados, según las normas ASTM D-120, IEEE 978 de 1984 [14] y otras afines, además de documentación científica [15] y de soporte académico [16].

\subsection{Aplicación del sistema de pruebas en guantes}

El sistema se probó en tres tipos de guantes: guantes de cocina, guantes de nitrilo para manejo de desechos tóxicos y guantes dieléctricos clase 00 , obteniendo los siguientes resultados:

Prueba de voltaje sostenido a guantes de caucho para cocina: la prueba se desarrolló como ensayo piloto, indicando un incremento en la corriente del lado de baja de hasta $980 \mathrm{~mA}$ (rms) con una tensión en el primario de $30 \mathrm{~V}$ ( $\mathrm{rms}$ ), lo que significó una potencia máxima de 29.4VA, que no implicó ningún riesgo para el transformador elevador.

El valor de impedancia del guante se obtuvo por la división entre la tensión medida y la corriente calculada en el lado de alta, encontrando una resistencia promedio de $12.6 \Omega$.

No se llevó el guante a la zona de ruptura, por lo que su resistencia permaneció constante (cercana a 12,6 $\Omega$ ). Lo cual se evidenció con el incremento de tensión y corriente, a medida que aumentaba el nivel de tensión de alimentación y la ausencia de fisuras o agujeros que permitieran que el agua pasara de un lado al otro. El guante de cocina no es elemento dieléctrico que permita su aplicación en TCT, ya que a pesar de soportar 92Vrms permitió la circulación de $7.316 \mathrm{~mA}$ eficaces, lo que implica riesgos para el trabajador.

El concepto final de la prueba dieléctrica es de rechazo, debido a que la estructura del caucho en este implemento no es pertinente en TCT y sus propiedades no permiten que la persona se aisle de manera segura.

Prueba de voltaje sostenido a guantes de nitrilo: con el fin de determinar la impedancia equivalente de otro tipo de guante no comercializado para TCT y establecer el funcionamiento del sistema con otros materiales, se hizo un ensayo con un guante de nitrilo conectado entre los terminales 1 y 2 . A partir de la medición de tensión y corriente en el lado de baja del transformador, se desarrolló la prueba hasta $60 \mathrm{~V}, 1,10 \mathrm{~A}$ lo cual implicó un nivel de potencia máximo de 66,1VA como parámetro seguro para la operación de dicho elemento. Con las mediciones directas de tensión y corriente en el guante se obtuvo un valor de aislamiento promedio de $29,6 \mathrm{k} \Omega$. 
Este ensayo permitió concluir que no se llevó el guante a la zona de ruptura, por lo que su resistencia de aislamiento permaneció constante (cercana a $29.6 \mathrm{k} \Omega$ ). Lo cual se evidenció con el incremento de tensión y corriente a medida que aumentaba el nivel de tensión de alimentación y la ausencia de fisuras o agujeros que permitieran que el agua pasara de un lado al otro.

El guante de nitrilo no es elemento dieléctrico que permita su aplicación en TCT, ya que a pesar de demostrar un nivel de impedancia alta, permitió la circulación de 14,09mA con un nivel de tensión de $412 \mathrm{Vrms}$. El concepto final de la prueba dieléctrica es de rechazo para TCT, puesto que sus propiedades de aislamiento no son seguras.

\section{Prueba de voltaje sostenido a guantes dieléctricos clase}

00: para este proyecto se contó con un par de guantes clase 00 , lo que permitió hacer un ensayo con el sistema de pruebas sobre un elemento real usado en TCT. Los guantes clase 00 son empleados para TCT a $500 \mathrm{~V}$ y deben someterse a pruebas de rigidez dieléctrica de $2500 \mathrm{~V}$ y de ruptura de $4000 \mathrm{~V}$

Se efectuó una prueba de tensión sostenida durante un minuto a un par de guantes clase 00 marca REGELTEX. En la Figura 6 se presenta la conexión de los elementos.
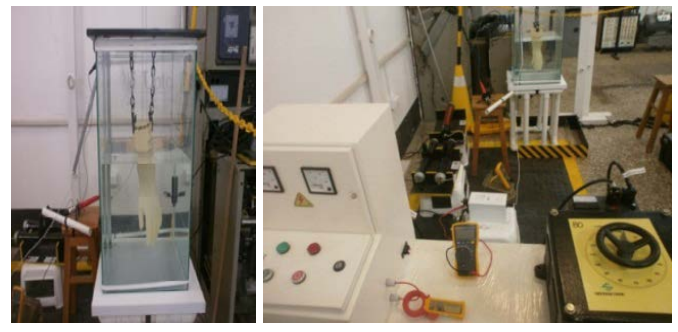

Figura 6. Prueba a guantes dieléctricos clase 00.

A partir de los valores medidos se estableció un flujo de corriente constante en baja tensión, con un valor cercano a las pruebas realizadas al transformador en vacío. De la medición directa de la corriente y la tensión en el guante se obtuvo una relación de la impedancia equivalente de dicho elemento con un valor cercano a $570 \mathrm{k} \Omega$.

Del ensayo se pudo concluir que el sistema de pruebas funcionó correctamente, al evidenciar la circulación de un nivel corriente en el lado de alta tensión cuando se cierra el circuito ente los terminales 1 y 2 con un elemento aislante cuyo equivalente resistivo fue de $570 \mathrm{k} \Omega$. La tensión aplicada al guante se incrementó hasta la tensión de prueba, permitiendo la circulación de 4,05mA con un voltaje de entrada de $12,2 \mathrm{~V}$. El aumento en la tensión, medido en el guante en función de la variación del voltaje de entrada, evidenció que el elemento se encontraba en condiciones tolerables de aislamiento.

El guante clase 00 probado sí es un elemento dieléctrico que permite su aplicación en TCT, demuestra un nivel de impedancia alta y permite la circulación de $4.05 \mathrm{~mA}$ con un nivel de tensión de $2480 \mathrm{Vrms}$. Por tanto, el concepto final de la prueba dieléctrica es de aceptación para TCT, ya que sus propiedades de aislamiento son seguras.

Tabla 4. Tabla resumen de la prueba a los tres tipos de guantes

\begin{tabular}{lllll}
\hline $\begin{array}{l}\text { Tipo de } \\
\text { guante }\end{array}$ & $\begin{array}{c}\text { Tensión } \\
\text { en alta }\end{array}$ & $\begin{array}{c}\text { Tensión } \\
\text { en baja }\end{array}$ & $\begin{array}{c}\text { Corriente } \\
\text { en alta }\end{array}$ & $\begin{array}{c}\text { Corriente } \\
\text { en baja }\end{array}$ \\
\hline $\begin{array}{l}\text { Guante } \\
\text { para } \\
\text { cocina }\end{array}$ & $92 \mathrm{~V}$ & $30 \mathrm{~V}$ & $7 \mathrm{~mA}$ & $1 \mathrm{~A}$ \\
\hline $\begin{array}{l}\text { Guante } \\
\text { de nitrilo }\end{array}$ & $412 \mathrm{~V}$ & $60 \mathrm{~V}$ & $14,09 \mathrm{~mA}$ & $1 \mathrm{~A}$ \\
\hline $\begin{array}{l}\text { Guante } \\
\text { clase } 00\end{array}$ & $2480 \mathrm{~V}$ & $12,2 \mathrm{~V}$ & $4,05 \mathrm{~mA}$ & $0,8 \mathrm{~A}$ \\
\hline
\end{tabular}

Los valores de tensión y corriente en los guantes de cocina y los de nitrilo no coinciden, porque existe un corto, debido a que ambos permiten el paso de corriente de su interior al exterior. Mientras que el guante dieléctrico clase 00 permitió tomar valores reales y datos acordes a lo que se exige en la norma.

Otra forma de verificar que se encontraba en corto la prueba, fue determinando la resistencia del guante, que para cada ensayo fue de $12,6 \Omega, 29,6 \mathrm{k} \Omega$ y $570 \mathrm{k} \Omega$, respectivamente. Se concluyó que el primer guante tiene una resistencia muy baja, pues permite que pasen niveles de corriente muy altas. El segundo tiene valores de resistencia mucho más altos, pero aún así, no son suficientes para proteger a una persona, mientras que los guantes dieléctricos son aptos para trabajo en redes energizadas, validando el sistema de pruebas y los valores de tensión que se permiten en la norma y por el fabricante.

\subsection{Aplicación del sistema de pruebas en pértigas}

Con el fin de verificar el funcionamiento del soporte construido para el anclaje de pértigas y tubos 
conductores, se llevó a cabo un ensayo preliminar con un tubo de PVC de ventilación de 1,5m de longitud, tal como se indica en la Figura 7.
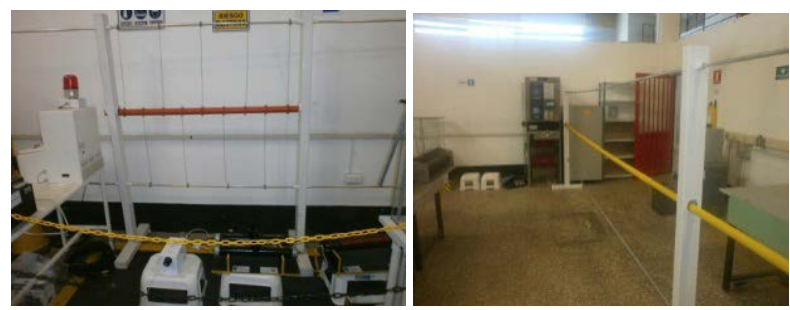

Figura 7. Prueba a tubo de PVC y pértiga de 7 secciones

Del ensayo se pudo concluir que el nivel de tensión aplicada sobre los terminales 1 y 2 durante un minuto llegó a 13010V. El aumento progresivo de la tensión sobre el tubo permitió verificar que el sistema no trabajó en una condición de cortocircuito, sino que funcionó correctamente, como se evidenció en la circulación de corriente en el lado de alta, cuando se cerró el circuito ente los terminales 1 y 2 con un elemento aislante, cuyo equivalente resistivo en promedio fue de $3,85 \mathrm{M} \Omega$, después de la estabilización del circuito.

La potencia del transformador elevador fue de máximo
42,3VA, obtenida por la multiplicación de la tensión y la corriente en el lado de baja. Valor cercano al $10 \%$ de máxima potencia permitida para dicho elemento, por lo que la prueba fue segura para el sistema.

Aunque los resultados del ensayo permiten apreciar el tubo de PVC como un elemento dieléctrico, su uso fue únicamente con fines demostrativos para la validación del sistema, pues dicho elemento no es aceptado para TCT en la técnica de distancia y, adicionalmente, la tensión máxima aplicada al elemento (13010V) es muy inferior a los $75 \mathrm{kV}$ establecidos como voltaje de prueba para pértigas comerciales.

Se calculó la relación de transformación empleando el método de los dos voltímetros, dando como resultado 125:1 cuando se obtiene un voltaje en el secundario similar al voltaje nominal para el cual fue fabricado el transformador; se pudo establecer un voltaje máximo de salida de $12600 \mathrm{~V}$ con un voltaje máximo de entrada al transformador de $109 \mathrm{~V}$, valor que debe ser considerado como el límite en la manipulación del variac por el operario del sistema de pruebas. La potencia máxima en el lado de baja es de 58 VA. Los datos de esta prueba se presentan en la Tabla 4 [19]

Tabla 5. Transformador en vacío

\begin{tabular}{|c|c|c|c|c|c|c|}
\hline Corriente & $\begin{array}{c}\text { Voltaje en } \\
\text { baja tensión } \\
\text { [Vrms] }\end{array}$ & $\begin{array}{c}\text { Alta tensión } \\
\text { con } \mathrm{R}=0 \\
{[\mathrm{Vrms}]}\end{array}$ & $\begin{array}{c}\text { Alta tensión } \\
\text { con } \mathrm{R}=5 \\
{[\mathrm{Vrms}]}\end{array}$ & $\begin{array}{c}\text { Alta tensión } \\
\text { con } \mathrm{R}=20 \\
{[\mathrm{Vrms}]}\end{array}$ & $\begin{array}{l}\text { Relación de } \\
\text { Transformación }\end{array}$ & $\begin{array}{c}\text { \% de error en relación de } \\
\text { transformación }\end{array}$ \\
\hline 0.21 & 0.3 & 21.9 & 18 & 17.3 & 73 & 41.6 \\
\hline 0.21 & 3.3 & 253 & 245 & 240 & 76.67 & 38.67 \\
\hline 0.21 & 4.94 & 392 & 386 & 380 & 79.35 & 36.52 \\
\hline 0.21 & 10.04 & 820 & 800 & 790 & 81.67 & 34.66 \\
\hline 0.22 & 15.04 & 1230 & 1220 & 1210 & 81.78 & 34.57 \\
\hline 0.22 & 19.57 & 1690 & 1660 & 1640 & 86.36 & 30.91 \\
\hline 0.22 & 25.06 & 2340 & 2192 & 2150 & 93.38 & 25.3 \\
\hline 0.26 & 49.77 & 5470 & 5132 & 5100 & 109.91 & 12.08 \\
\hline 0.27 & 55.38 & 6180 & 5900 & 5820 & 111.59 & 10.73 \\
\hline 0.28 & 59.68 & 6770 & 6538 & 6470 & 113.44 & 9.25 \\
\hline 0.29 & 65.86 & 7530 & 7200 & 7070 & 114.33 & 8.53 \\
\hline 0.3 & 69.9 & 8060 & 7890 & 7660 & 115.31 & 7.75 \\
\hline 0.32 & 75.8 & 8780 & 8350 & 8310 & 115.83 & 7.34 \\
\hline 0.34 & 80.9 & 9230 & 8870 & 8820 & 114.09 & 8.73 \\
\hline 0.36 & 85.5 & 9771 & 9620 & 9560 & 114.28 & 8.58 \\
\hline 0.39 & 90.3 & 10270 & 10200 & 10180 & 113.73 & 9.01 \\
\hline 0.42 & 95.6 & 11010 & 10925 & 10750 & 115.17 & 7.87 \\
\hline 0.45 & 100.6 & 11600 & 11480 & 11350 & 115.31 & 7.75 \\
\hline 0.49 & 104.9 & 12400 & 11950 & 11820 & 118.21 & 5.43 \\
\hline 0.53 & 109.52 & 12600 & 12490 & 12420 & 115.05 & 7.96 \\
\hline
\end{tabular}


El dato de la corriente muestra el consumo del variac y el transformador elevador. Cuando se mida la corriente de fuga en las pruebas, es necesario descontar este valor de corriente de vacío del variac y del transformador elevador. La relación de transformación presenta una desviación con niveles de tensión bajos, pero se van disminuyendo al momento de subir el voltaje. De acuerdo con lo anterior, se recomienda medir la tensión de prueba directamente en el lado de AT.

Con el fin de resaltar las prácticas en los diferentes ambientes de estudio, se implementan las pruebas de tensión aplicada a elementos de protección personal, con la presencia de alumnos de la UPTC, Facultad Seccional Duitama, fortaleciendo la investigación y el desarrollo, con base en la enseñanza de proyectos propios en curso y terminados, tal como se hace en estudiantes de colegios, optando por determinar estrategias, uso de herramientas pedagógicas y prácticas para lograr facilitar la comprensión del tema [20], permitiendo programar y determinar el comportamiento de los ensayos por medio de software y programas computacionales que facilitan el diseño experimental y análisis de datos y resultados como se indica en determinación de parámetros de un robot móvil de lego mindstorms ${ }^{\circledR}$ [21] que promueve la interacción de los datos, la simulación y la realidad, para concluir cuales son los puntos mínimos, máximos y medios de estudio.

\section{CONCLUSIONES}

El campo de aplicación de los TCT se divide en tres partes importantes, que permiten el desarrollo de las actividades de mantenimiento y ampliación de redes energizadas capaces de brindar un óptimo servicio al usuario, como son el conocimiento de los trabajos, una destreza por parte de los operarios y la inclusión de este proyecto en el buen funcionamiento de los elementos de protección personal, que certifiquen el uso de manera confiable y eficiente, cumpliendo con los requerimientos legales.

Los ensayos desarrollados validan el sistema propuesto desde el punto de vista mecánico, teniendo en cuenta los materiales seleccionados y dimensiones de las estructuras construidas para los elementos de soporte y ubicación de guantes o pértigas. Desde el punto de vista eléctrico, se pudieron realizar ensayos para establecer capacidades dieléctricas cuando el sistema funcionaba en condiciones de circuito abierto, con carga resistiva o en corto circuito entre los terminales de ubicación del elemento de prueba. Se verificó además el cumplimiento de la normatividad establecida bajo las secuencias de prueba, el cumplimiento de parámetros de seguridad para el uso del sistema y el seguimiento a los ensayos hechos, mediante el uso del sistema de gestión para la generación de conceptos de aprobación o rechazo de los elementos probados.

Como resultado de este trabajo, la Universidad Pedagógica y Tecnológica de Colombia, Facultad seccional Duitama, cuenta con un montaje para realizar pruebas dieléctricas a materiales y equipos eléctricos hasta $15 \mathrm{kV}$, siendo este el primer paso para la creación de un laboratorio de pruebas electromecánicas en la región de Boyacá y Casanare. Este laboratorio podrá prestar servicios de extensión, investigación y docencia.

\section{AGRADECIMIENTOS}

Los autores agradecen a la Escuela de Ingeniería Electromecánica, al Centro de Investigaciones CIFAD y a la Dirección de Investigaciones DIN de la Universidad Pedagógica y Tecnológica de Colombia UPTC. La adquisición de materiales y equipos necesarios para el sistema de prueba fue financiada por los recursos asignados al proyecto de investigación "Evaluación del estado de guantes y pértigas dieléctricas empleados para trabajos en redes energizadas de la Empresa de Energía de Boyacá" SGI 1744, a través de la participación en la convocatoria de la Dirección de Investigaciones DIN capital semilla 2015; así como los equipos de medición y generación de tensión disponibles en el grupo GridsE y en el Laboratorio de Electricidad de la UPTC, Facultad Seccional Duitama.

\section{REFERENCIAS}

[1] Ministerio de Minas y Energía, Reglamento Técnico de Instalaciones eléctricas RETIE. Bogotá: MME, 2015.

[2] Comisión de Regulación de Energía y Gas CREG. (2016). Resolución CREG-070 de 1998, CREG 179 de 2014 y CREG 024 de 2016 Asignación de obligaciones de energía firme del cargo por confiabilidad. [En línea]. Disponible: http://www.creg.gov. co/cxc/secciones/documentos/resoluciones.htm.

[3] Ministerio de la Protección Social, de Colombia. (2009). Resolución 001348 de 2009. [En línea]. Dis- 
ponible: http://www.alcaldiabogota.gov.co/sisjur/ normas/Norma1.jsp?i=36213.

[4] Organismo Nacional de Acreditación-ONAC. (s.f.). [En línea]. Disponible en http://www.onac.org.co/

[5] Norma Técnica Colombiana NTC-ISO/IEC 17025. Requisitos generales para la competencia de los laboratorios de ensayo y calibración.

[6] ASTM D 120. (s.f.). Standard specification for rubber gloves. Disponible en los formatos ASTM. O [En línea]. Disponible: http://www.astm.org.

[7] UNE-EN 60903:2005. (2005). Trabajos en tensión. Guantes de material aislante. [En línea]. Disponible: http://www.insht.es/InshtWeb/Contenidos/Documentacion/TextosOnline/Divulgacion_Normativa/Ficheros/FDN_20.pdf.

[8] Matrimol S.A.S. (s.f.). Nombre de pruebas en matrimol, Universidad del Valle. [En línea]. http://matrimol.com/pruebaslab.php.

[9] IEEE 978 de 1984, "Guide for in service maintenance and electrical testing of line lives tools", 1984.

[10] ASTM F711 (s.f.). Standar specification for in- service care of Insulating Gloves and Sleeves. [En línea]. Disponible: http://www.astm.org

[11] IEC 855 de 1985, "Insulatingfoam-filled tubes \& solid rods for live working", 1985.

[12] IEC 832 de 1988, "Insulting poles (insulatin sticks) \& universal tool attachements for live work", 1988.

[13] IEC 410 de 1973.

[14] IEEE 978 de 1984, "Guide for in-service maintenance and electrical testing of live-line tools", 1984.
[15] N. Kolcio y R.A. Peszlen, "Electrical Aspects of Testing Insulating Gloves", IEEE Transactions on Power Apparatus and Systems, vol.PAS-102, n.으 7, p. 2364-2368, Feb. 26 2007. DOI: 10.1109/ TPAS.1983.318163.

[16] H. Ospina, "Control predictivo para la detección de alarmas en pruebas de aislamiento de Pértigas utilizadas en redes de distribución", Revista Energía y Computación, vol. 17 n.ㅇ 1, págs.5-6, 2009.

[17] M. Balpinarli, J.J. Dai y G. Gela, "AC and DC breakdown versus thickness characteristics for rubber gloves", IEEE transactions on power delivery, vol. 3 n.o 1, pp. 384-391, 1988.

[18] M. S. A. A. Hammam, N. Yoshimura, W. Brockway, G. Adams, A. Fini and H. Nowak, "Puncture breakdown characteristics of protected rubber insulating gloves", IEEE transactions on power delivery, vol. 5 n.ㅇ 2, pp. 538-547, 1990.

[19] C.E. Pinto-Salamanca, Diseño y montaje de un sistema de pruebas dieléctricas en guantes y pértigas hasta $15 \mathrm{kV}$. Duitama: libro, resultado de trabajo de grado, 2016.

[20] M. Pinto, "Uso de la robótica educativa como herramienta en los procesos de enseñanza", Ingeniería Investigación y Desarrollo, vol. 10 n. 1, pág. 16-20, enero-junio de 2010

[21] M. Pinto, "Determinación de parámetros de un robot móvil de lego mindstorms ${ }^{\circledR ", ~ I n g e n i e r i ́ a ~ I n-~}$ vestigación y Desarrollo , vol. 5 n.ㅇ 2, págs. 10-12, julio-diciembre de 2007. 